10-2002

\title{
An Intelligent System for Monitoring the Microgravity Environment Quality On-Board the International Space Station
}

\author{
Paul P. Lin \\ Cleveland State University, p.lin@csuohio.edu \\ Kenol Jules \\ NASA Glenn Research Center
}

Follow this and additional works at: https://engagedscholarship.csuohio.edu/enme_facpub

Part of the Computer and Systems Architecture Commons, and the Space Vehicles Commons How does access to this work benefit you? Let us know!

\section{Publisher's Statement}

(C) 2002 IEEE. Personal use of this material is permitted. Permission from IEEE must be obtained for all other uses, in any current or future media, including reprinting/republishing this material for advertising or promotional purposes, creating new collective works, for resale or redistribution to servers or lists, or reuse of any copyrighted component of this work in other works.

\section{Original Citation}

Lin, P. P., \& Jules, K. (2002). An intelligent system for monitoring the microgravity environment quality onboard the International Space Station. leee Transactions on Instrumentation and Measurement, 51, 5, 1002-1009.

This Article is brought to you for free and open access by the Mechanical Engineering Department at EngagedScholarship@CSU. It has been accepted for inclusion in Mechanical Engineering Faculty Publications by an authorized administrator of EngagedScholarship@CSU. For more information, please contact library.es@csuohio.edu. 


\title{
An Intelligent System for Monitoring the Microgravity Environment Quality On-Board the International Space Station
}

\author{
Paul P. Lin and Kenol Jules
}

\begin{abstract}
An intelligent system for monitoring the microgravity environment quality on-board the International Space Station is presented. The monitoring system uses a new approach combining Kohonen's self-organizing feature map, learning vector quantization, and a back propagation neural network to recognize and classify the known and unknown patterns. Finally, fuzzy logic is used to assess the level of confidence associated with each vibrating source activation detected by the system.
\end{abstract}

Index Terms-Adaptive pattern recognition and classification (APRC), back propagation neural network (BPNN), learning vector quantization (LVQ), microgravity, self-organizing feature map (SOFM), source detection, system monitoring.

\section{INTRODUCTION}

$\mathbf{S}$ TARTING with Flight 6A (STS-100) in April 2001, the International Space Station (ISS) became scientifically operational. It provides the scientific community with much longer periods of microgravity condition compared to the U.S. Space Shuttle. The Principal Investigator Microgravity Services (PIMS), part of the Microgravity Measurement and Analysis Project (MMAP) at the NASA Glenn Research Center (GRC), has the responsibility for measuring, analyzing and characterizing the microgravity environment on-board the ISS, since many of the experiments conducted on the ISS require the knowledge of the microgravity environment quality for accurate analysis of the science experimental data.

The main objective of this work is to develop an intelligent monitoring system, which not only can classify incoming signals into known patterns, but also identify the unknown ones, in near real time. Since the ISS is being built in increments, its fundamental frequency will change some until assembly is complete. Thus, identifying the unknown patterns is as important as the known ones. At this point, there are five sensors that can simultaneously receive acceleration data. The monitoring system is fully automated from analyzing the sensor data to making the final decision as to what vibrating sources are active, with some degree of confidence.

P. P. Lin is with the Mechanical Engineering Department, Cleveland State University, Cleveland, OH 44115-2425 USA (e-mail: p.lin@csuohio.edu).

K. Jules is with the NASA Glenn Research Center, Cleveland, OH 44135 USA (e-mail: kenol.jules@grc.nasa.gov).

\section{INTELLIGENT MONITORING SYSTEM}

Currently, the acceleration data analysis and interpretation to characterize the Space Shuttle and other spacecraft platforms microgravity environment is performed by a PIMS data analyst. The acceleration data received from the sensors are in the time domain. They are, then, transformed to the frequency domain by means of a fast Fourier transform (FFT), from which the power spectral density (PSD) is generated. The PSD is a function that quantifies the distribution of energy in a signal with respect to frequency and it is used to identify and quantify vibratory (oscillatory) components of the acceleration environment. The major peak values of the PSDs must be associated with the fundamental or natural frequencies of different vibrating sources. Such analysis is time consuming. To ease the analyst's work, it is desirable to automate the analysis process described above. Also, automation will provide space-experiment principal investigators (PIs) on-line access to the acceleration data via the PIMS world wide web site, where they can see what vibrating sources are active in near real time, which might impact their experiments.

The intelligent monitoring system is designed to perform the following four tasks:

1) Detect the current vibrating sources on-board the ISS in near real time (source detection).

2) Classify known patterns (pattern classification).

3) Recognize unknown patterns (pattern recognition).

4) Assess the level of confidence associated with each vibrating source activation (confidence determination).

The schematic diagram of the overall monitoring system is shown in Fig. 1 and described in detail below.

\section{A. Source Detection}

In terms of source detection, the system must automatically detect the fundamental frequencies of the vibratory disturbance sources from the acceleration data measured by the accelerometers (sensors) located at different locations on-board the ISS. The fundamental frequencies correspond to the major peaks of the PSD data. First of all, based on the degree of PSD data variation, the entire frequency spectrum is automatically divided into several regions. Within each region, all data points are fitted with a high-order curve. A data point whose function value (the PSD value, in this case) is significantly (such as three times) higher than the fitted value and whose sign of gradient changes from positive to negative, is considered as a major peak. 

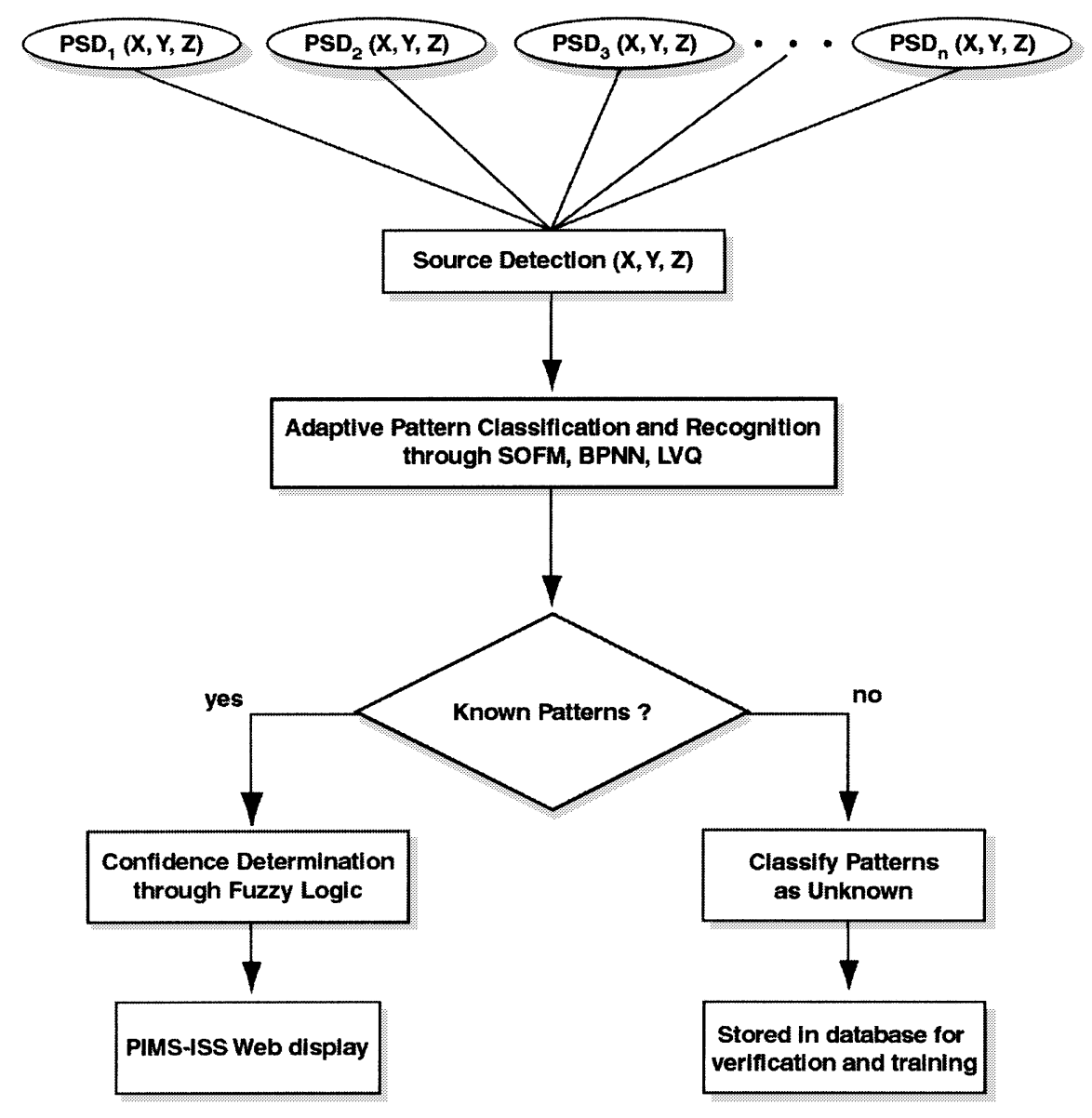

Fig. 1. Overall monitoring system.

\section{B. Pattern Classification}

On-board the ISS, there are many disturbance sources, such as fans, pumps, life support systems, etc. In addition, disturbances can be generated during vehicle docking. As a result, the structural modes of the ISS change due to the addition of the new ISS modules. For the purpose of source classification, these disturbance sources need to be identified as soon as they are detected. The Kohonen's self-organizing feature map (SOFM) [1] is used to cluster the known patterns. A known pattern consists of the nominal values of the previously measured frequency and acceleration of an existing disturbance source. SOFM is a special class of artificial neural networks. It is based on competitive learning in which the output neurons compete among themselves to be activated or fired and the winner takes it all. Furthermore, SOFM is characterized by the formation of a topological map of the input patterns in an unsupervised manner. The topological map allows one to visualize the order of organized input patterns in the input space.

The classification approach used in this work consists of cluster and class (pattern) grouping. A cluster is a group of data with the same classification features. In this case, a cluster represents a group of measured frequency and acceleration values of a single vibrating source and the mean value of this group is called the cluster center. Thus, each cluster center contains a pair of data representing the nominal fundamental frequency and the nominal acceleration values of a known vibrating source. A class is formed by grouping several clusters that share the same attributes into a group. In other words, class is one level higher than cluster. Since the ISS has multiple degrees of freedom, it possesses multiple fundamental frequencies, known as structural modes. In this case, several clusters represent the structural modes of the ISS. These several clusters form a class. Likewise, the harmonics of a vibrating source, which by themselves are clusters, also form a class. Grouping clusters into classes is accomplished by using learning vector quantization (LVQ) [2], [3], which is a supervised learning technique. The strength of LVQ networks is that they can be trained to recognize classes made up of multiple unconnected regions, which cannot be accomplished by SOFM. A multiple-unconnected-region is referred to a class that contains both the fundamental frequency of a vibrating source and its related harmonics. The aforementioned ISS structural modes and their harmonics are a typical example of such multiple-unconnected-region. The LVQ offers the advantage of grouping several clusters into the same class (same source, in this case).

\section{Pattern Recognition}

To prevent possible misclassification, the classified patterns need to be verified. For each known pattern, the allowable tolerance (deviation) ranges from the nominal values of frequency and acceleration are specified. Thus, as soon as an input pattern 
is assigned to a cluster, a verification process begins by checking if the pattern falls within the maximum allowable range [The maximum allowable range is knowledge based. For example, the $\mathrm{Ku}$ band antenna, used by the Shuttle for communication and data downlink to ground, has a disturbance signature around $17 \mathrm{~Hz}$ with associated acceleration magnitude level between 100 to $300 \mu \mathrm{g}$ (RMS). Knowing such range from past data, an allowable deviation range from the nominal value is specified, for example, $\pm 5 \%$ based on past observation]. Therefore, a pattern, which has been classified and verified, is recognized as a known pattern. On the other hand, any pattern that falls outside of the allowable range is recognized as an unknown pattern (meaning that the system has not seen it before or been trained yet to recognize it).

The pattern recognition is accomplished by building two separate filter masks for frequency and acceleration. Each mask can perform instant filtering by means of neural network mapping. The mapping is accomplished by using another class of artificial neural networks, called the back-propagation neural network (BPNN) [4], which uses supervised learning rules. A BPNN based on a Gaussian distribution with respect to the nominal value of any known pattern has been trained. The distribution is bounded by three standard deviations $( \pm 3 \sigma)$. Therefore, if a frequency value with a $\pm 5 \%$ deviation from the nominal frequency of a vibrating source of interest is specified, the deviation is equivalent to $( \pm 3 \sigma)$, likewise, for acceleration. It is worthwhile to note that the BPNN was trained in terms of the unit of $\sigma$, which is dimensionless. Therefore, there is only one trained BPNN for both frequency and acceleration.

To recognize a pattern, the BPNN generates the so-called degree of belongingness (DOB) between 0 and 1 for both frequency and acceleration. For instance, a value below 0.1 (using $3 \sigma$ ) for either frequency or acceleration means that the detected source does not belong to the cluster and is recognized as an unknown pattern. On the other hand, if the detected frequency is exactly the same as the nominal frequency, then the DOB value of frequency will be 1 , likewise, for acceleration.

\section{Confidence Determination}

The objective here is to provide an index, which gives a relative assessment as to how confident the monitoring system is regarding the determination of which source is active at any moment in time.

On-board the ISS, there are many accelerometers with different sampling rates. They may be moved to different locations from time to time and may or may not be located in the scientific racks where the experiments are located. Therefore, the locations of known sources, sensors and racks should be known by the system. Such information is used to design the decisionmaking process, which in turn generates the confidence index.

It is very possible that the same disturbance source is detected by more than one sensor. In this case, it is desired to determine which sensor is most relevant to a specific experiment. Instead of classifying the relevance as relevant or irrelevant, it is quantified using the concept of partial truth. As a result, the degree of relevance (DOR) is between 0 and 1 , where 0 and 1 mean very irrelevant and very relevant, respectively. The DOR between sen-
TABLE I

ACCURACY COMPARISON FOR THE FIRST DATA SET

\begin{tabular}{l|l|l|l|l}
\hline PSD & $\begin{array}{l}\text { Frequency } \\
\text { Data }\end{array}$ & $\begin{array}{l}\text { Estimated RMS } \\
\text { Resolution }\end{array}$ & $\begin{array}{l}\text { Actual RMS } \\
\text { Acceleration }\end{array}$ & $\begin{array}{l}\text { Difference } \\
\%\end{array}$ \\
\hline Pxx & $0.0305 \mathrm{~Hz}$ & 1.9 milli-g & 1.9 milli-g & 0 \\
\hline Pyy & $0.0305 \mathrm{~Hz}$ & 4.9 milli-g & 4.8 milli-g & 2.1 \\
\hline Pzz & $0.0305 \mathrm{~Hz}$ & 1.0 milli-g & 1.0 milli-g & 0 \\
\hline
\end{tabular}

TABLE II

ACCURACY COMPARISON FOR THE SECOND DATA SET

\begin{tabular}{l|l|l|l|l}
\hline $\begin{array}{l}\text { PSD } \\
\text { Data }\end{array}$ & $\begin{array}{l}\text { Frequency } \\
\text { Resolution }\end{array}$ & $\begin{array}{l}\text { Estimated RMS } \\
\text { Acceleration }\end{array}$ & $\begin{array}{l}\text { Actual RMS } \\
\text { Acceleration }\end{array}$ & $\begin{array}{l}\text { Difference } \\
\%\end{array}$ \\
\hline Pxx & $0.0610 \mathrm{~Hz}$ & 2.8 milli-g & 2.7 milli-g & 3.7 \\
\hline Pyy & $0.0610 \mathrm{~Hz}$ & 1.4 milli-g & 1.4 milli-g & 0 \\
\hline Pzz & $0.0610 \mathrm{~Hz}$ & 0.68 milli-g & 0.68 milli-g & 0 \\
\hline
\end{tabular}

Note: The above-estimated RMS acceleration values were calculated using the proposed procedure based on the PSD data at $60.18 \mathrm{~Hz}$.

sors and experiment racks greatly depends on their geometric relationship.

To accomplish this, fuzzy logic [5] is used since it is suitable for dealing with imprecision and uncertainty. Fuzzy logic measures the truth of a given situation as a matter of degree. Between the input and the output, there is a black box that does the work through the use of if-then rules. The input for the fuzzy logic contains membership functions of each input variable and the output also contains membership functions of each output variable. In this work, there are three input membership functions: the DOB of frequency, the DOB of acceleration and the DOR of sensors with respect to experiment racks. The DOB and DOR values are both between 0 and 1 . The output membership function of fuzzy logic is the degree of confidence (DOC), which is also between 0 and 1, where 1 represents $100 \%$ confidence that a source of interest is active and 0 means that the source is not. An example of a fuzzy logic rule for a sensor is: if DOB is high and DOR is high, then the DOC is high.

\section{TECHNICAL NOVELTIES}

In the course of developing the monitoring system, many technical problems arose, but were overcome. Below we briefly discuss how the process leads to some technical innovations (novelties) in the field of pattern classification.

\section{A. Generating Additional Dimension for Pattern Classification}

Generally speaking, the more dimensions used in pattern classification, the better the classification will be. This is simply because each pattern will have more distinct features. In this work, however, once the acceleration data have been transformed from the time domain to the frequency domain through FFT, it is difficult to relate a detected fundamental frequency magnitude level in the frequency domain to its corresponding acceleration in the time domain. Such task is time consuming and resource intensive in terms of storing and tracking data in the two domains.

In the time domain, an acceleration magnitude value is the combined effect of all vibrating sources at that instant of time. Therefore, the acceleration values in the time domain can not be used to identify which vibrating sources are active. Conse- 


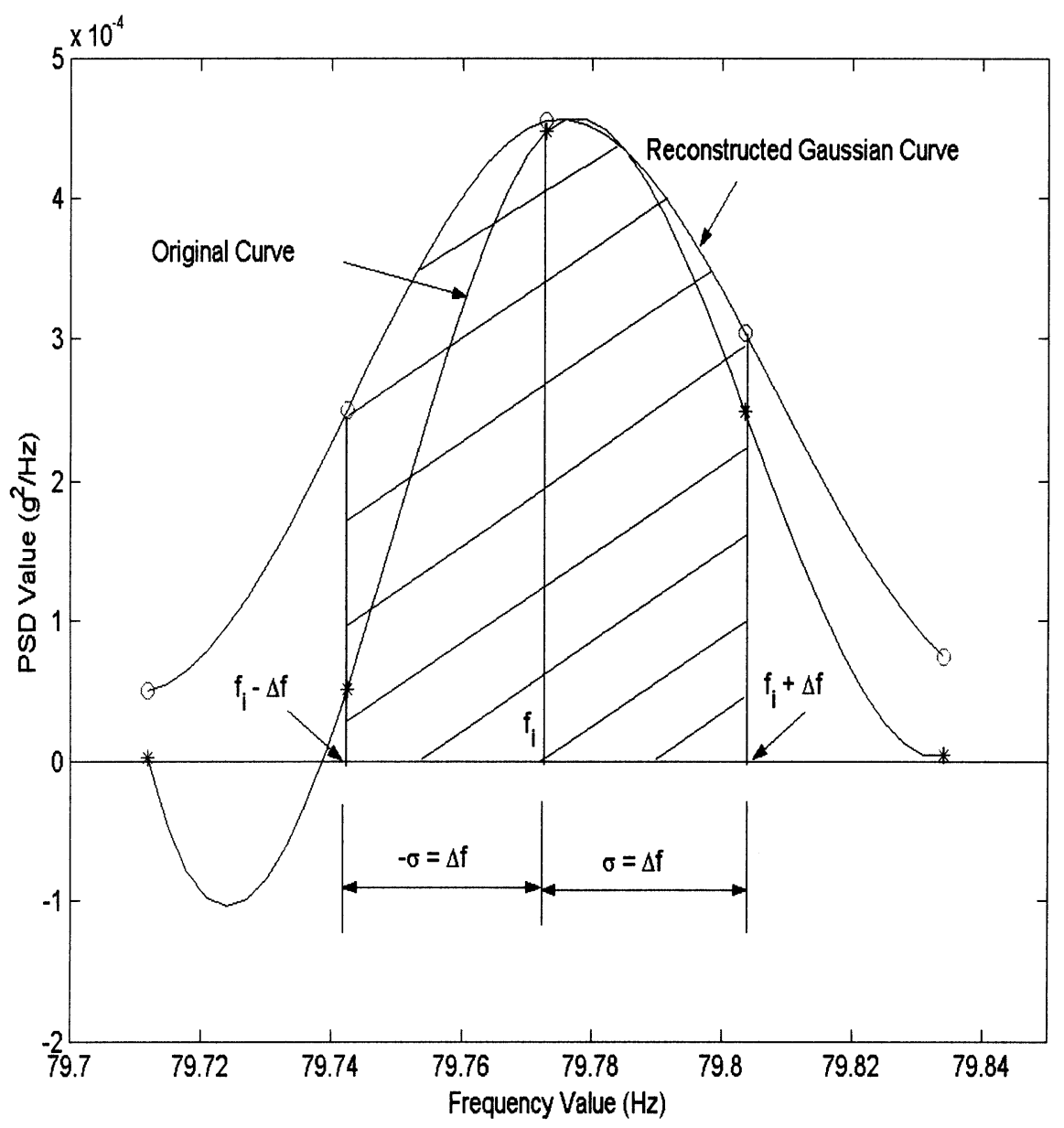

Fig. 2. Integration of PSD Data with respect to Frequency.

quently, source detection has to be made in the frequency domain. However, it is necessary to know the corresponding acceleration value for each detected frequency in the frequency domain. To do so, one of Parseval theorems [6] is used. The theorem states that the total energy may be determined either by computing the energy per unit time and integrating over all time or by computing the energy per unit frequency and integrating over all frequencies. The theorem further states that there exists an equivalence between the root mean square (RMS) value of a signal computed in the time domain to that in the frequency domain. The equivalent RMS acceleration can be calculated as follows

$$
A_{\mathrm{RMS}}=\left[\sum_{i=0}^{k} p(i) \Delta f\right]^{1 / 2}
$$

where $k=0,1,2 .,(n / 2), n$ is the number of samples in the time domain, $p(i)$ is the PSD value at frequency $f(i)$ and $\Delta f$ is the frequency resolution.

Furthermore, this theorem can be used to attribute a fraction of the total energy in a signal to a user-specified band of frequencies by appropriately choosing the limits of integration. However, the theorem does not address what the appropriate limits of integration are. In this paper, a procedure for quantifying the RMS acceleration, which addresses the choice of the limits of integration, is developed. It is described below.
Step 1) The PSD data around the frequency of interest are reconstructed by a Gaussian distribution to minimize the measurement noise. Conceptually, the standard deviation $(\sigma)$ value of this distribution should be relatively small in order to make a narrow band around the frequency of interest. The $\sigma$ value was determined by simulations using some sets of previous Space Shuttle mission data in the frequency domain and the time domain. For each data set, the error between the estimated RMS acceleration from the frequency domain and the actual RMS acceleration from the time domain was compared while varying the $\sigma$ value. As a result of the simulations, it was found that setting the $\sigma$ value equal to $\Delta f$ yields the smallest error. The accuracy comparisons are shown in Tables I and II.

Step 2) The reconstructed PSD data are integrated with respect to frequency from $f_{i}-\Delta f$ to $f_{i}+\Delta f$, where $f_{i}$ and $\Delta f$ stand for the frequency value of interest and the PSD frequency resolution, respectively. Such integration is essentially equivalent to the hatched area shown in Fig. 2. Note that the integration limits were determined by the simulations mentioned above.

Step 3) The square root of the integrated result is taken. As a result, the time-domain equivalent RMS acceleration $(g)$ for the frequency of interest is recovered. 
This procedure was verified using two sets of the Space Shuttle mission data in the frequency domain and the time domain (for comparison). The accuracy of the acceleration estimation for each set is given in Tables I and II.

Here Pxx, Pyy, and $P z z$ are the PSD data in the $x, y$ and $z$ axes, respectively. Note that the above-estimated RMS acceleration values were calculated using the proposed procedure based on the PSD data at $79.77 \mathrm{~Hz}$, whereas the actual RMS acceleration values came from the Space Shuttle past mission data collected from sensors in the time domain.

Generally speaking, the actual acceleration magnitude measured in the time domain is the combined acceleration of all vibrating sources at that time. However, it is possible to find a vibrating source that happens to be the only active source at some instant of time. Such sources can be found in the frequency domain by identifying the dominant PSD value at some specific frequency such as $79.77 \mathrm{~Hz}$ or $60.18 \mathrm{~Hz}$, in this case. As shown in the above tables, the estimation errors are quite small. This procedure was implemented for this work. As a result, each detected fundamental frequency is accompanied by the estimated RMS acceleration magnitude to form a pair of data to be used for the pattern classification.

\section{B. Proper Weighting With Multiple Dimensions}

The SOFM uses Euclidean distance to measure the distance between an input pattern and the cluster center of interest. For example, the Euclidean distance in two-dimensional space is defined as

$$
D=\left[\left(x_{1}-x_{1, c}\right)^{2}+\left(x_{2}-x_{2, c}\right)^{2}\right]^{1 / 2}
$$

where $x_{1}$ and $x_{2}$ are the values of the input pattern in dimensions 1 and 2, respectively and $x_{1, c}$ and $x_{2, c}$ are the cluster centers in dimensions 1 and 2 , respectively. In this work, the two dimensions are the frequency and acceleration magnitude. Therefore, the Euclidean distance of an input pattern $(f, a)$ to a cluster center $\left(f_{c}, a_{c}\right)$ can be expressed as

$$
D=\left[\left(f-f_{c}\right)^{2}+\left(a-a_{c}\right)^{2}\right]^{1 / 2}=\left(\Delta f^{2}+\Delta a^{2}\right)^{1 / 2} .
$$

Since SOFM uses Euclidean distance for classification, improper weighting between these two dimensions could lead to misclassification. For example, consider the following two cluster centers, whose units are $\mathrm{Hz}$ and $\mathrm{g}$ (Table III).

If a source is detected at $71.8 \mathrm{~Hz}$ and 46 micro-g, then without proper scaling the data point will be classified into cluster $n+1$ because the first dimension (frequency, in this case) is much more dominant than the second dimension (acceleration). This results in the shortest Euclidean distance between the detected source and cluster $n+1$ [see (3)]. In this case, the Euclidean equation degenerates from 2-D to 1-D. However, if a scaling factor of $2 \times 10^{5}$ were applied to the second dimension (i.e., the values of acceleration are multiplied by this factor in order to generate an equally weighted scale to preserve the two dimensionality of the data), then the same source would be classified into cluster $n$, which is correct because the Euclidean distance is the shortest and both dimensions are equally weighted. It is very important to make $\Delta f$ and $\Delta a$ [as shown in (3)] the same order of magnitude. Otherwise, one dimension alone will
TABLE III

Cluster Centers and Their Dimensional Ranges

\begin{tabular}{l|l|l|l}
\hline $\begin{array}{l}\text { Cluster } \\
\text { No. }\end{array}$ & $\begin{array}{l}\text { Cluster } \\
\text { center }\end{array}$ & $\begin{array}{l}\text { Range for the } \\
1 \text { st dimension }\end{array}$ & $\begin{array}{l}\text { Range for the } \\
2^{\text {nd }} \text { dimension }\end{array}$ \\
\hline $\mathrm{n}$ & $\left(71,50^{*}\right)$ & $69.6-72.4$ & $40-60$ \\
\hline $\mathrm{n}+1$ & $\left(72,40^{*}\right)$ & $70.6-73.4$ & $20-60$ \\
\hline \multicolumn{4}{|c}{ Where ${ }^{*}$ denotes multiplication of $10^{-6}$}
\end{tabular}

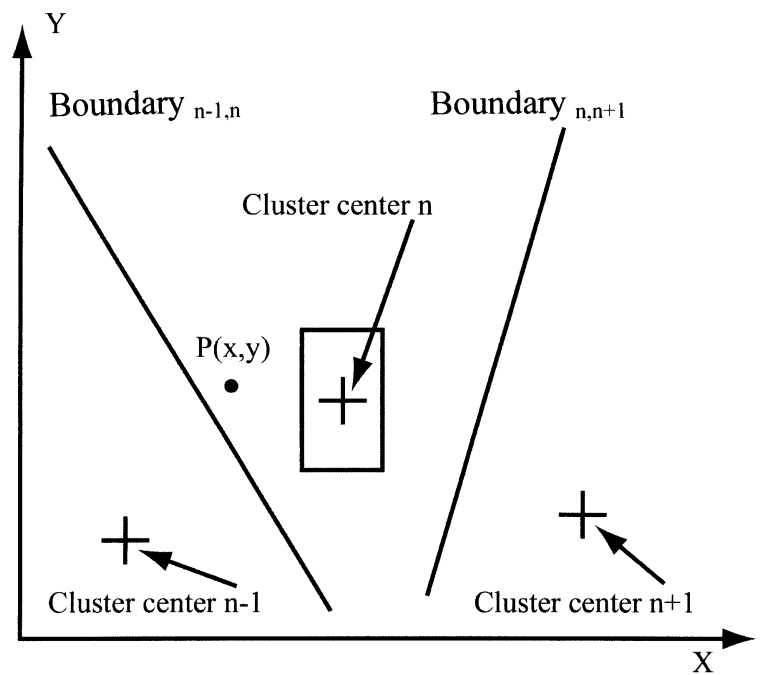

Fig. 3. Cluster centers and their boundaries.

dictate the selection of the cluster, which will result in pattern misclassification.

\section{Modified Model for Pattern Classification and Recognition}

The SOFM classifies every input data point into one of the established cluster centers. By default, the boundary between any two adjacent clusters is essentially located in the middle of the two cluster centers (see Boundary ${ }_{n-1, n}$ - the boundary between clusters $n-1$ and $n$ and Boundary ${ }_{n, n+1}$ - the boundary between clusters $n$ and $n+1$, Fig. 3 ).

Therefore, any point, such as $P(x, y)$ (marked by “॰”) that falls within the region between boundary ${ }_{n-1, n}$ and boundary $_{n, n+1}$ belongs to cluster $n$.

Let us suppose that cluster $n$ has a range in each dimension, as enclosed by the rectangle around the cluster center (see Fig. 3). Any point that falls outside the rectangular region, but still between boundary ${ }_{n-1, n}$ and boundary ${ }_{n, n+1}$, should not be classified into cluster $n$. In fact, it should be classified as an unknown pattern. Unfortunately, SOFM has no such ability. Lippman [7] proposed an approach to combine SOFM and LVQ in order to place the input vectors into the desired classes. His approach enhanced the capability of pattern classification. However, it still can not recognize unknown or new patterns. To address these shortcomings, the hybrid model is proposed in this paper. This model, as shown in Fig. 4, combining SOFM, BPNN, and LVQ, is referred to herein as adaptive pattern recognition and classification (APRC).

In this proposed model, the BPNN is inserted in between SOFM and LVQ for unknown pattern recognition, while SOFM and LVQ are used solely for the classification of known patterns. 


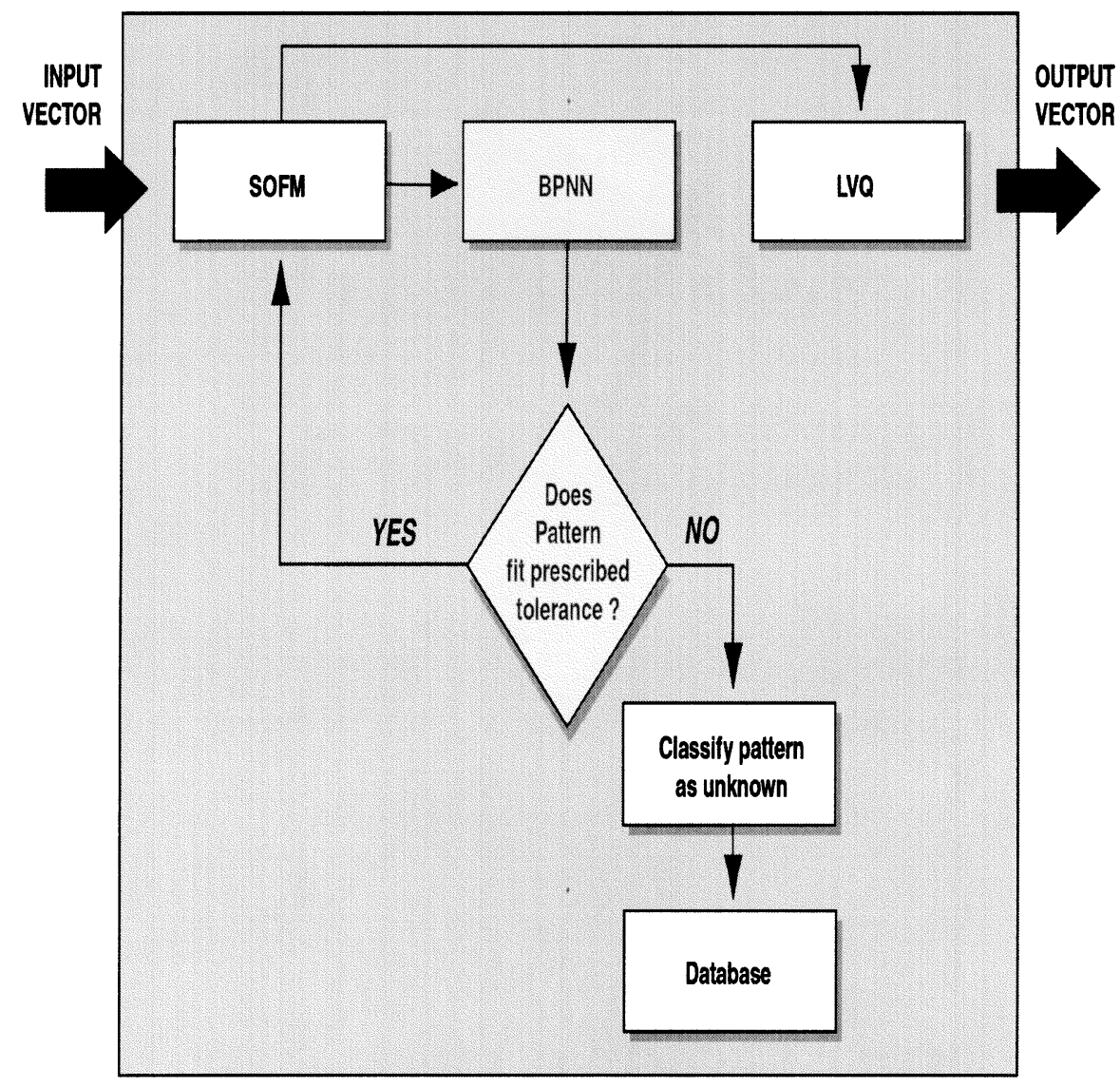

Fig. 4. Adaptive pattern recognition and classification.

\section{Taking Into Account Multi-Dimensional Ranges of Neighboring Clusters}

In multi-dimensional space, each cluster may have a different range in each dimension, as shown in Fig. 5. In this case, the Kohonen's SOFM [1] will classify the data point $P(x, y)$ into cluster $n$ due to the shortest Euclidean distance between the point and the center of cluster $n$ (even though that data point belongs to cluster $n-1)$. However, in the APRC model, since the point falls outside the specified range of each dimension of cluster $n$, that data point will be placed on hold until the ranges of the neighboring cluster (cluster $n-1$, for instance) are checked. As a result, the data point will be classified into cluster $n-1$ as a known pattern. Without the multi-dimensional neighboring cluster checking feature of APRC, the data point would have been classified as an unknown pattern, which would have been incorrect. The proposed APRC model has the ability to avoid such possible misclassification in multi-dimensional space for clusters with cross-boundary range overlapping.

In multidimensional space, this type of misclassification could occur even with proper scaling among dimensions. The problem is essentially due to the different dimensional ranges for each cluster when two cluster centers are close to each other. The only remedy to this problem is to check the neighboring clusters in each dimension. In this monitoring system, neighboring cluster checking was implemented using the BPNN, which compares every unknown pattern with the neighbors of the rejected cluster to make sure that the unknown

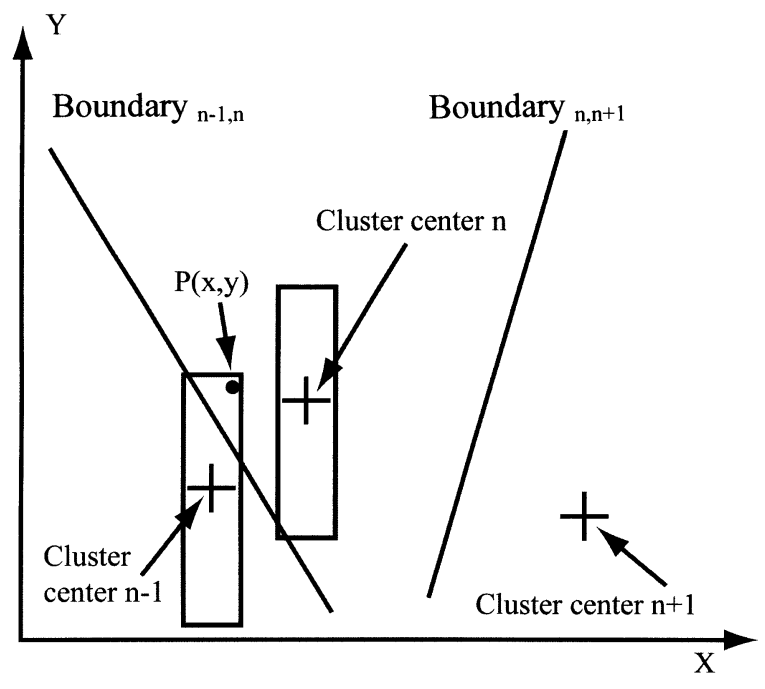

Fig. 5. Multi-dimensional ranges for each cluster.

pattern, in fact, does not belong to any of the surrounding clusters.

In summary, the proposed APRC used in this work is superior to Lippman's model [7] in the following aspects:

a) It can recognize unknown patterns.

b) It can avoid pattern misclassification.

c) It takes into account multi-dimensional ranges of neighboring clusters. 


\section{THE APRC APPROACH}

Fig. 4 shows the schematic diagram of the APRC approach. The procedure of the approach is described below in detail. The program begins by retrieving PSD data sets generated from the real-time acceleration data downlinked from the ISS to perform peak detection. For each detected relevant peak, the program uses the modified Parseval theorem to estimate the RMS acceleration, from which the acceleration magnitude level from the time domain is calculated, for each detected frequency. For each pair of acquired parameter detected (frequency and acceleration), the program uses SOFM to screen each set by assigning it to some potential cluster (remember that SOFM uses the Euclidean distance for classification; thus, if it is used alone, it could lead to pattern misclassification).

To overcome the weakness of SOFM, the program then uses the BPNN to check if the detected pair falls within a prescribed range (for both frequency and acceleration). The BPNN either affirms or rejects the preliminary clustering made by SOFM. If it affirms it, the pair is left in the assigned cluster. Otherwise, the BPNN performs the neighboring-cluster check. If a match is found, the pair is removed from the preliminary assigned cluster and reassigned to the new cluster by SOFM. If no match is found, the cluster is removed from the previously assigned cluster and transferred to a database reserved for unknown patterns for further analysis and possible training. Once the pair is affirmed, SOFM sends it to LVQ, which classifies the pair as well as matching the value (frequency and acceleration) of the pair with the name of the pattern, for example, fan or pump, in the known database. Once, the name of the pair is identified, the vibrating source name along with its value is sent to the PIMS web site for display and viewing by principal investigator teams.

\section{Simulation Result}

At the time when this simulation was performed, no real-time acceleration data was available from the ISS. Therefore, the monitoring system was simulated using two sets of data from previous Space Shuttle missions and two sets of data from previous NASA missions on the Russian MIR Space Station. For these four sets of data, the program correctly detected the fundamental frequencies of the vibratory disturbance sources, recognized and classified them into the right clusters and classes.

The result of the simulation is discussed in detail below. For the simulation a database was created containing 43 clusters simulating known patterns to the system and 15 classes simulating the vibrating classes to which the 43 clusters belonged. The simulation started with peak detection of all the three axes PSD data generated from the four previous missions mentioned above. Taking the $X$-axis PSD data as an example, in the range of 0 to $200 \mathrm{~Hz}, 58$ peaks (clusters) were detected. Out of these 58 peaks, the program recognized 24 as known patterns and 34 as unknown. The reason a large number of unknowns were detected is due to the fact that the trained patterns (stored in the database as known patterns) were mostly between 0 and $100 \mathrm{~Hz}$. Only three known patterns were over $100 \mathrm{~Hz}$ in the database.

As soon as a peak from the PSD data was detected, the modified Parseval theorem was used to calculate the actual accel-
TABLE IV

EXAMPLE OF NEIGHBORING CLUSTERS

\begin{tabular}{l|c|c|c}
\hline $\begin{array}{l}\text { Cluster } \\
\text { No. }\end{array}$ & $\begin{array}{l}\text { Nominal } \\
\text { Frequency }(\mathrm{Hz})\end{array}$ & $\begin{array}{l}\text { Maximum } \\
\text { Acc. }(\mu \mathrm{g})\end{array}$ & $\begin{array}{l}\text { Minimum } \\
\text { Acc. }(\mu \mathrm{g})\end{array}$ \\
\hline 29 & 71 & 50 & 40 \\
\hline 30 & 71 & 20 & 10 \\
\hline 31 & 72 & 40 & 20 \\
\hline
\end{tabular}

eration magnitude associated with that peak. For example, a peak was detected at $38.0859 \mathrm{~Hz}$ and the acceleration magnitude was calculated to be $11.32 \mu \mathrm{g}$. The SOFM temporarily assigned the pattern in cluster 17 , which has the prescribed range of $38 \pm 5 \%$ for frequency and 10 to $30 \mu \mathrm{g}$ for acceleration. The SOFM passed the values of the detected peak to the BPNN for verification in order to avoid possible misclassification. The BPNN compared the frequency and acceleration values with the nominal values of cluster $17(38 \mathrm{~Hz}$ and $20 \mu \mathrm{g}$, respectively) and determined the DOB for frequency and acceleration as 0.912 and 0.034 , respectively. In this case, the frequency of $38.0859 \mathrm{~Hz}$ was very close to the nominal value, while the acceleration of $11.32 \mu \mathrm{g}$ was just slightly above the minimum acceleration $10 \mu \mathrm{g}$. This pattern was confirmed and then sent back to SOFM for final clustering. Since both frequency and acceleration values were within the prescribed ranges, that pattern was recognized as a known pattern belonging to cluster 17. Finally, SOFM passed that information to LVQ, which determines which class that pattern belongs to and its actual name. In this case, it was the signature of a fan associated with an experiment called glovebox.

The following illustrates how the program was able to prevent pattern misclassification. Let us examine the three known patterns in the neighborhood of 71 and $72 \mathrm{~Hz}$, shown in Table IV.

The program detected a relevant peak at $71.2585 \mathrm{~Hz}$ with calculated acceleration of $38.6 \mu \mathrm{g}$. Initially, this pattern was temporarily identified as an unknown pattern because it was compared with cluster 29 . The program then checked the first neighboring cluster (cluster 30), but the pattern was again rejected because its acceleration was beyond the prescribed acceleration range of cluster 30 . The program continued to check the second neighboring cluster (cluster 31) and successfully recognized the pattern as a known pattern (cluster 31). The reason cluster 29 was picked as the right match at the first pass is because SOFM used Euclidean distance for clustering. If only SOFM were used, the pattern would have been assigned to cluster 29, which would have been the wrong cluster, but since the BPNN was used to check the prescribed ranges as well as neighboring clusters, two mistakes were avoided. First, a pattern misclassification was avoided (cluster 31 instead of 29). Second, instead of classifying the pattern as an unknown pattern, it was recognized as a known one due to the neighboring cluster checking capability of the program.

For this simulation, the total CPU time from peak detection to pattern recognition and classification was about 4 seconds for each axis using a PC with $500 \mathrm{MHz}$ clock speed. The simulation result was verified by examining the corresponding color spectrograms in $x, y$ and $z$-axes, respectively. A spectrogram is a three-dimensional plot that shows PSD values (represented by 
colors) versus frequency versus time. It is primarily for the purpose of visualization. The result showed a $100 \%$ success rate in recognizing and classifying the detected frequencies and acceleration magnitudes into known and unknown patterns. In this simulation, the degree of relevance for each sensor to any specific experiment rack was not tested.

\section{CONCLUSIONS}

The monitoring system discussed in this paper has demonstrated its capability to automatically detect the vibratory disturbance sources and to correctly identify and classify them. The APRC approach presented here has the ability to recognize and classify known and unknown patterns, as well as to prevent possible pattern misclassification. A procedure to quantify the RMS acceleration in the frequency domain, which allows for the calculation of the acceleration magnitude levels in the time domain, was developed. The acceleration magnitude calculation gives SOFM an extra dimension, which lessens to some degree the potential of pattern misclassification. Fuzzy logic is used to exploit the tolerance for imprecision, uncertainty and partial truth, along with the experience of the human experts (by means of fuzzy logic rules), to make intelligent decisions as to what vibrating sources are more relevant to a specific sensor.

Our experience is that it takes a data analyst quite some time to analyze a typical set of data, such as 4,193 points, to identify the known and unknown patterns in three axes. This type of work is considered post data analysis. In contrast, the proposed monitoring system can automatically retrieve PSD data and perform data analysis in near real time. More importantly, the recognized events (i.e., known patterns) are instantly posted on the web site of the NASA PIMS so that the space-experiment principal investigators around the world can see what vibrating sources are active at any time.

Currently, there are six sensors installed inside the International Space Station to measure the acceleration in the vibratory regime $(0.01-300 \mathrm{~Hz})$. They usually reside at certain fixed locations for some time and could measure the acceleration of the same vibrating source at the same time. The locations of most vibrating sources, such as fans and pumps, are known. Future work includes developing a multi-sensor fusion scheme to automatically choose the sensor that is most relevant to a specific source.

\section{ACKNOWLEDGMENT}

The authors would like to thank K. Hrovat, PIMS Data Analyst of ZIN Technologies, for the knowledge and insights he pro- vided throughout this work, T. A. Reckart of ZIN Technologies, who did the graphic works, and K. McPherson and D. Francisco of the Microgravity Science Division, NASA Glenn Research Center.

\section{REFERENCES}

[1] T. Kohonen, Self-Organization and Associative Memory, 3rd ed. New York, Berlin, Germany: Springer-Verlag, 1988.

[2] — , "Learning Vector Quantization for Pattern Recognition," Helsinki Univ. Technol., Finland, Tech. Rep. TTK-F-A601, 1986.

[3] — , "Improved versions of learning vector quantization," in Int. Joint Conf. Neural Networks, vol. 1, San Diego, CA, 1990, pp. 545-550.

[4] M. T. Hagan, H. B. Demuth, and M. Beale, Neural Network Design. Boston, MA: PWS, 1996.

[5] C. T. Lin and G. C. S. Lee, Neural Fuzzy Systems. Englewood Cliffs, NJ: Prentice-Hall, 1996.

[6] A. V. Oppenheim, A. S. Willsky, and S. H. Nawab, Signals \& Systems, 2nd ed. Englewood Cliffs, NJ: Prentice-Hall, 1997, p. 312.

[7] R. P. Lippmann, "Pattern classification using neural networks," IEEE Commun. Mag., vol. 27, pp. 47-64, 1989. 